\title{
Miniaturized load sensor using quartz crystal resonator constructed through microfabrication and bonding
}

\author{
Yuichi Murozaki, Kousuke Nogawa and Fumihito Arai*
}

\begin{abstract}
Highly sensitive, wide-measurement-range compact load sensors are desirable for various applications, including measurement of biosignals, manipulation and stiffness measurement of cells, and so on. Conventional load sensors are highly sensitive but have relatively small measurement ranges. A load sensor using an AT-cut quartz crystal resonator (QCR) has superior characteristics such as, high accuracy, improved strength under compressive stress, long-term stability, and compact size. However, a retention mechanism is required to firmly support the QCR because the QCR is easily broken by stretching and bending motions. Conventional machining processes are not suitable for further miniaturization of the sensor. Even if the retention mechanism were miniaturized, the assembly process is complicated.

In this paper, we propose a novel design and fabrication method for a load sensor using the QCR. Using microfabrication and bonding, the assembly process was simplified. We demonstrate the feasibility of a miniaturized QCR load sensor whose volume is $24.6 \mathrm{~mm}^{3}$ (width is $4 \mathrm{~mm}$, height is $5.6 \mathrm{~mm}$, depth is $1.1 \mathrm{~mm}$ ). The experimental results showed that the nonlinearity and hysteresis were $0.94 \%$ F.S. and $1.68 \%$ F.S., respectively. Additionally, sensitivity of the sensor was $1458 \mathrm{~Hz} / \mathrm{N}$. We improved the sensitivity and stability of the sensor; the fluctuation was $0.04 \mathrm{mN}$ over a period of $1 \mathrm{~min}$. Moreover, the effects of the temperature change were evaluated. The temperature and the sensor output were linear within the range of $20^{\circ} \mathrm{C}-50^{\circ} \mathrm{C}$.
\end{abstract}

Keywords: Quartz crystal resonator; Load sensor; Microfabrication

\section{Background}

Highly sensitive, wide-measurement-range, compact load sensors are desirable in many fields, such as the medical field and biological fields. Using a high-performance load sensor, we can realize health management by simultaneous detection of multiple biosignals, such as distribution of body (high load), pulse/blood pressure, and body motion by breath (small loads) on a bed/chair. Moreover, it could be possible to handle cells without damage and to measure the stiffness of the cells using the load sensor for force feedback in manipulation. Load sensors have employed different methods, such as the use of strain gauges $[1,2]$, piezoelectric vibration [3,4], and capacitance changes [5]. Conventional load sensors can be

\footnotetext{
* Correspondence: arai@mech.nagoya-u.ac.jp

Department of Micro-Nano Systems Engineering, Nagoya University, Nagoya,
} Japan

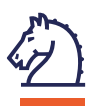

highly sensitive; however, in most cases, their measurement ranges are relatively narrow.

To realize a high-performance load sensor with both high sensitivity and a wide measurement range, we focused on the quartz crystal resonator (QCR). We developed a load sensor using the QCR (QCR load sensor). Quartz crystal is a piezoelectric element. The QCR generates a periodic signal with high stability based on vibration. The resonant frequency of a QCR changes with high linearity depending on the external force [6,7]. In addition, quartz crystals have high strength under compressive stresses [8]. Therefore, a high-resolution and wide-measurement-range load sensor can be realized using the QCR.

However, the QCR is fragile and easily broken by stretching and bending [9]. Therefore, a retention mechanism is required to affix the QCR to avoid detrimental horizontal forces [10-14]. Moreover, the retention mechanism 
plays an important role in efficiently transmitting external loads to the QCR. Fabrication of the retention mechanism and assembly process are complicated; miniaturization of the retention mechanism is quite difficult. Additionally, the sensor output is not linear owing to a gap between the retention mechanism and the QCR, and therefore, preload must be applied to the QCR to obtain linear output. In our previous work, a screw mechanism was used to apply preload to the retention mechanism $[13,14]$. This increased the number of sensor parts and further complicated assembly. In addition, to achieve further miniaturization and improvements in the sensor sensitivity, conventional machining processes are not feasible owing to the small size of the sensor elements.

In this paper, we aim to improve the QCR load sensor with regard to miniaturization and sensitivity to increase its number of effective applications. We propose a novel design and fabrication method of the load sensor using microfabrication and bonding. In this study, the sensor was successfully miniaturized and its sensitivity was improved.

\section{Methods}

\section{Conceptual design of QCR load sensor}

Figure 1 shows the concept for the miniaturized QCR load sensor made by microfabrication and bonding. Smaller structures can be fabricated by microfabrication. We used only three parts, and therefore, the assembly process was simplified. The parts are made by microfabrication and are bonded together (Figure 1). The QCR is fixed between two retention parts with leaf spring structures. Because of the gapless design, the previously used screw mechanism used to induce preload was not necessary. Without it, the number of the parts required was decreased and the assembly simplified. In addition, because each part is flat, we were able to employ microfablication to make the fine parts.

Sensor output and the QCR frequency shift depend on the load applied to the QCR. External loads compress both the retention mechanism and the QCR element.

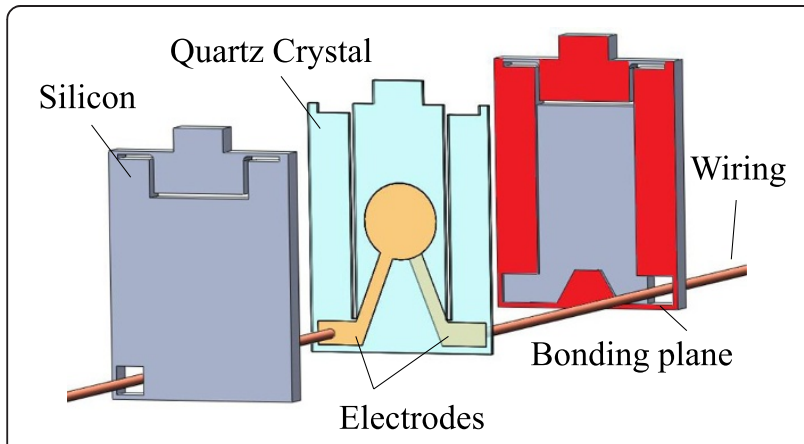

Figure 1 Concept of the miniaturized QCR load sensor made by microfabrication and bonding.
Therefore, we defined the ratio of the compressive load applied to the QCR $\left(F_{q}\right)$ divided by the vertical component of external force (load, $F_{v}$ ) as "load transfer efficiency $(\eta)$ " by the following:

$$
\eta=\frac{F_{q}}{F_{v}}
$$

The frequency shift $(\Delta f)$ is proportional to the compressive load applied to the QCR $\left(F_{q}\right)$, and we referred to this proportionality constant as "load sensitivity $\left(S_{l}\right)$ ".

$$
S_{l}=\frac{\Delta_{f}}{F_{q}}
$$

Considering the load transfer efficiency $(\eta)$, the sensor sensitivity $\left(S_{S}\right)$ is given by the following equation:

$$
S_{s}=\frac{\Delta_{f}}{F_{v}}=\eta \cdot S_{l}
$$

Here, load sensitivity $\left(S_{l}\right)$ is constant and depends on the QCR material properties (cross sectional area, temperature, cut direction, etc.). Therefore, to increase the sensor sensitivity, it is necessary to improve the load transfer efficiency. The load transfer efficiency is improved if the rigidity of the leaf spring in the vertical direction is low. However, it is difficult to miniaturize the leaf spring further using conventional machining processes. Therefore, we used microfabrication to miniaturize the leaf spring $[15,16]$.

\section{QCR design}

An AT-cut quartz crystal has superior temperature stability at room temperature $[17,18]$. When AC voltage is applied between two metal electrodes on both sides of the AT-cut QCR, thickness-shear vibration is generated along the quartz crystal's electrical axis ( $x$-axis).

Therefore, we designed the shape of the AT-cut QCR as shown in Figure 2. The bonding plane and oscillation component of the QCR are separated by a slit. The load is applied in the direction $34.8^{\circ}$ from the $x$-axis of the AT-cut QCR. Load sensitivity is not a function of temperature fluctuations in this direction [19]. Stable vibration of the QCR is necessary to obtain highly stable sensor outputs; the QCR oscillates both mechanically and electrically. Vibration is generated from the center of the electrode of the QCR, and bonding to the surface will disturb the vibration of the QCR. Therefore, the bonding plane is kept at a distance from the electrode. Thus, the oscillation component of the QCR was designed with a rectangular cross section.

\section{Retention mechanism design}

A schematic of the retention mechanism is illustrated in Figure 3. The retention part is made by processing a 


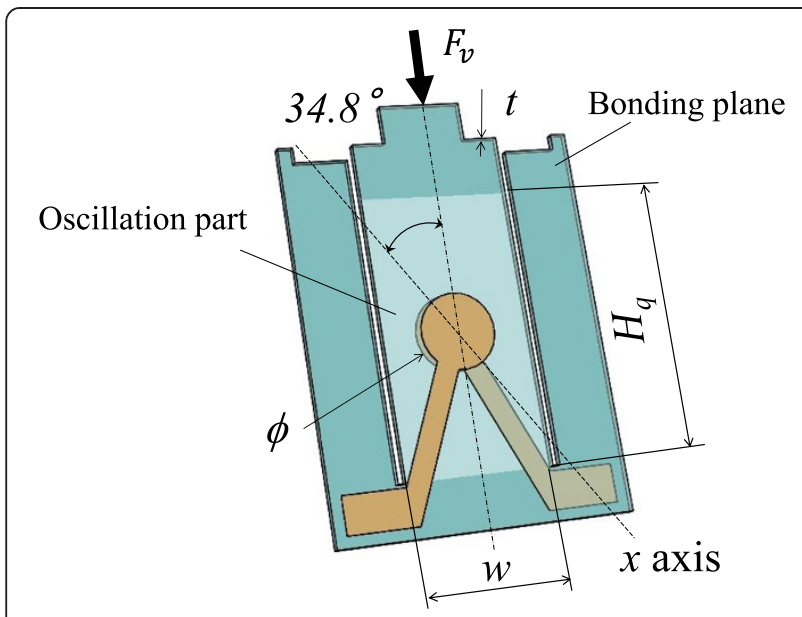

Figure 2 Schematic of the QCR.

single silicon $(\mathrm{Si})$ wafer. The height of the leaf spring must be small to improve the sensor sensitivity. Therefore, we employed microfabrication techniques to fabricate the fine retention parts.

One surface of each retention part is slightly etched not to interfere with the oscillation and wiring components. The retention parts have a hole for wiring connections. Figure 4 shows the analytical model of the sensor where the total structure is regarded as the combination of the springs. C-part and V-part indicate the ideal contact parts between the center structure of the QCR and the leaf spring and between the leaf spring and the flame of the sensor, respectively. The parameters $k_{q}, k_{b} k_{s}, R_{q}$ and $R_{s}$ show the spring constants of the center structure of the QCR, the leaf spring, and the frame structure of the sensor, and reaction force to the center structure of the QCR, and to the frame structure of the sensor, respectively. From the calculation of equilibrium of force at the C-part and V-parts, the following equation was derived:

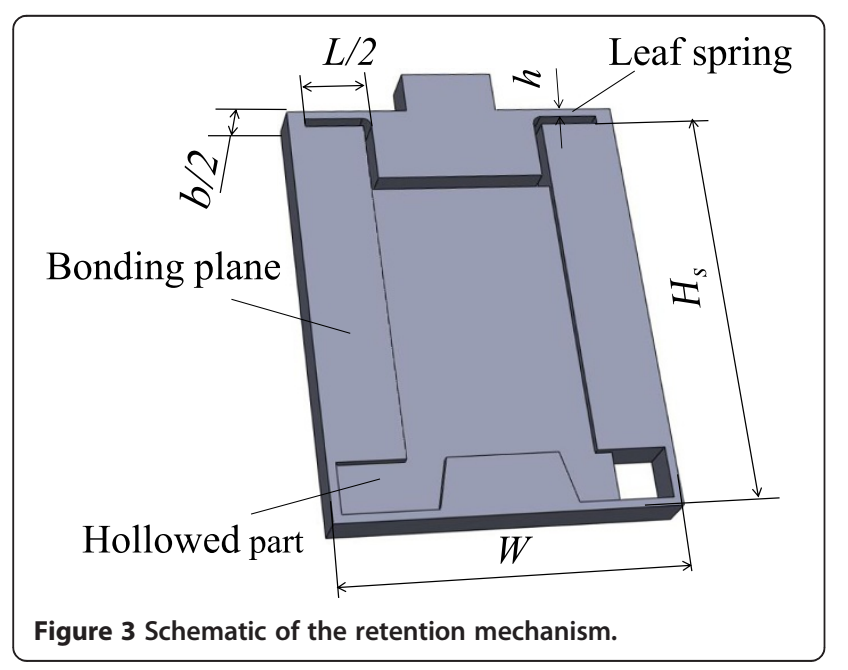

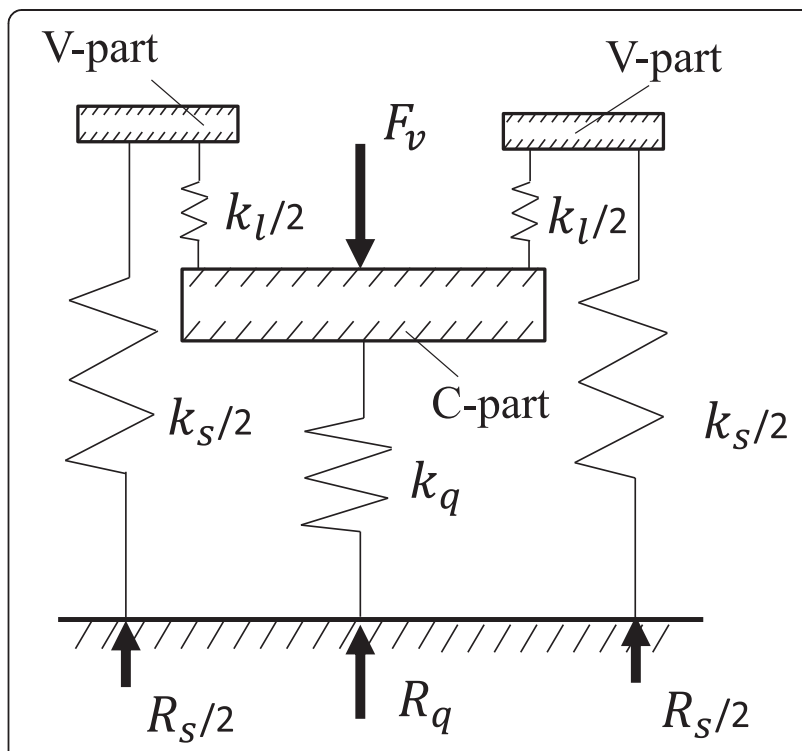

Figure 4 Dynamical model of the sensor.

$$
F_{v}=\left(\frac{k_{s}}{K_{q}} \frac{k_{l}}{k_{s}+k_{l}}+1\right) \cdot R_{q}
$$

Here, $R_{q}$ is the reaction force of $F_{q}$ in Eq. (2). Thus, the load transfer efficiency $(\eta)$ is derived from Eqs. (2)-(4) as follows.

$$
\eta=\frac{k_{q}\left(k_{s}+k_{l}\right)}{k_{s} k_{l}+k_{q} k_{s}+k_{l} k_{q}}=\frac{1+k_{l} / k_{s}}{k_{l} / k_{q}+1+k_{l} / k_{s}}
$$

Here, $k_{q}$ and $k_{s}$ are given by

$$
\begin{aligned}
& k_{q}=\frac{w t \cdot E_{q}}{H_{q}} \\
& k_{s}=\frac{W b \cdot E_{s}}{H_{s}}
\end{aligned}
$$

where $E_{q}$ is the Young's modulus of the quartz crystal and

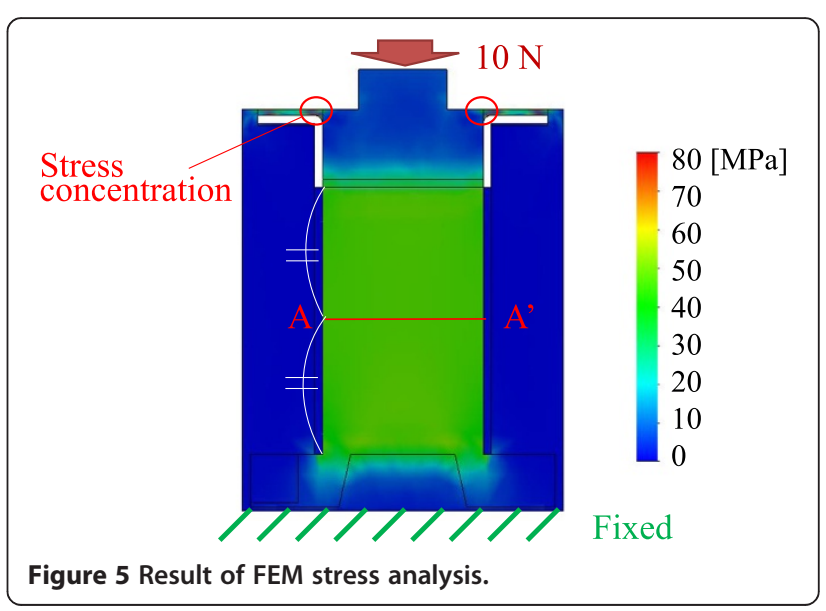




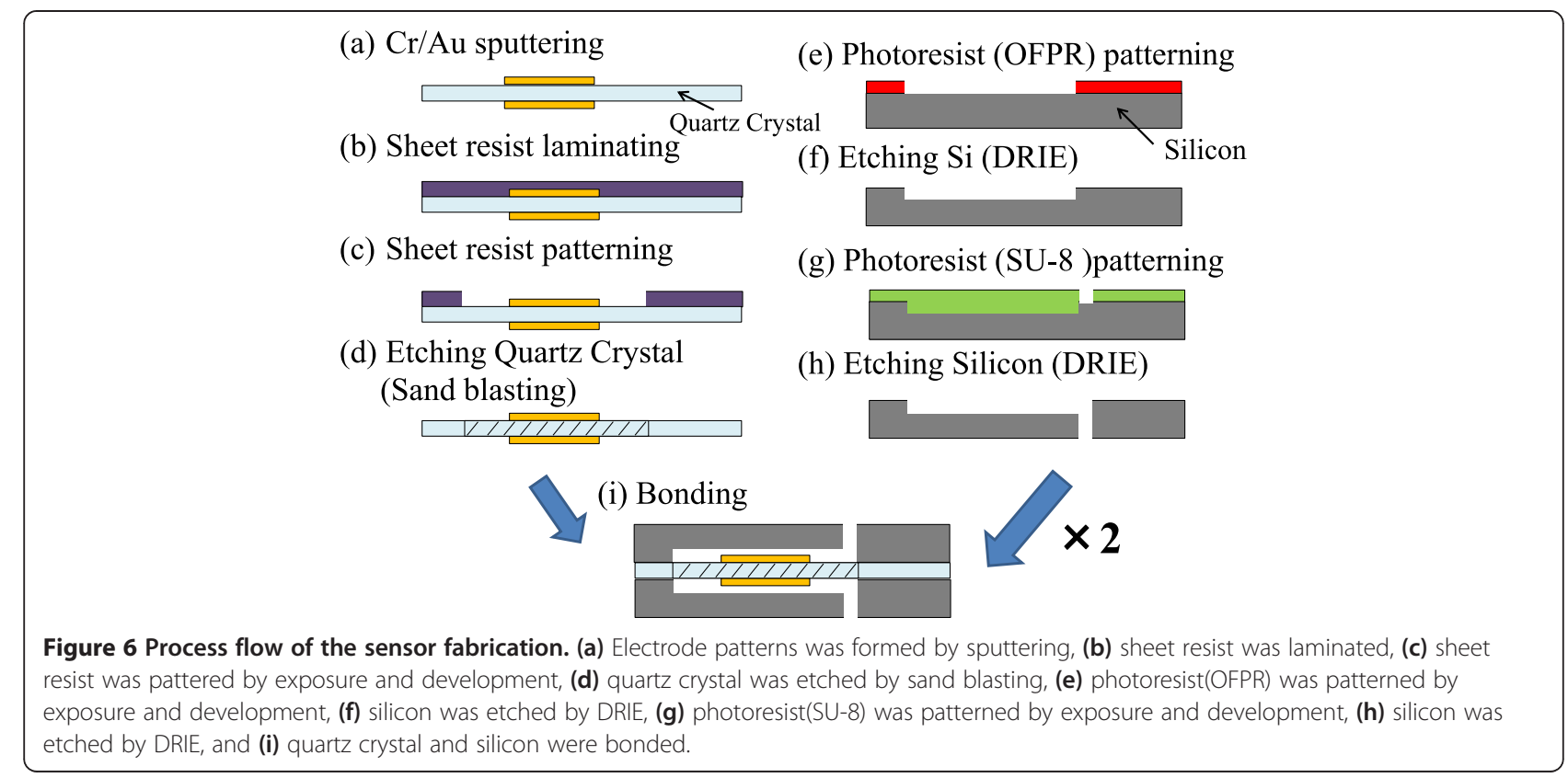

$E_{s}$ is the Young's modulus of Si. We assumed that both ends of the leaf spring are supported by the beam with a rectangular cross section (width is $b$, height is $h$, and length is $l$ ). Therefore, $k_{l}$ is given by the following equation:

$$
k_{l}=\frac{16 E_{s} b h^{3}}{L}
$$

$k_{l}$ must be less than $k_{q}$, to increase the load transfer efficiency ( $\eta$ ), which is from Eq. (5). From Eq. (8), it is evident that high load transfer efficiencies can be realized by decreasing $h$. However, $b$ must be sufficiently large to firmly support the QCR. Therefore, the ideal leaf spring has a large aspect ratio.

Here, we determined the dimensions of the QCR and the retention parts via calculation using Eqs. (5)-(8) such that the load transfer efficiency $(\eta)$ is $0.950: w=2.0 \mathrm{~mm}$,

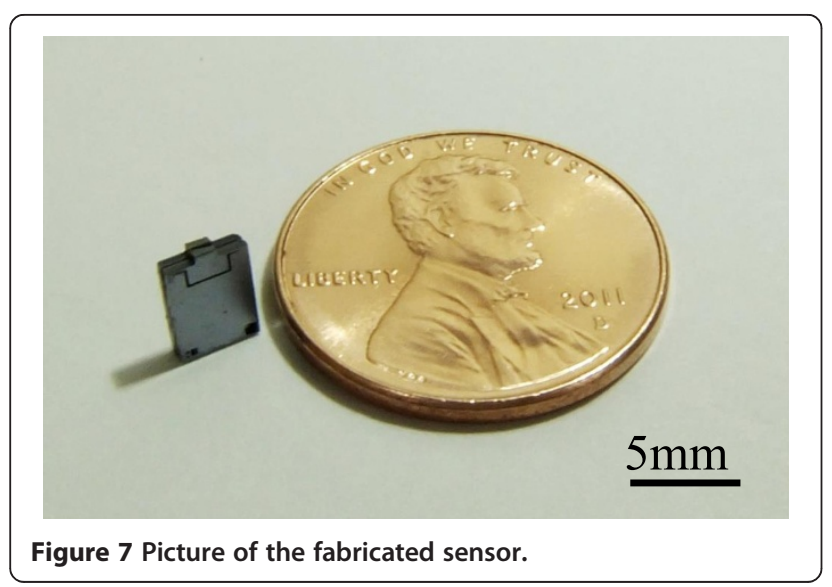

$H_{q}=3.5 \mathrm{~mm}, t=0.1 \mathrm{~mm}, W=4.0 \mathrm{~mm}, H_{s}=5.0 \mathrm{~mm}$, $b=1 \mathrm{~mm}, h=0.69 \mathrm{~mm}$, and $L=1.6 \mathrm{~mm}$.

In practice, the base of the leaf spring was rounded to prevent stress concentration. We calculated load transfer efficiency using finite element analysis with SolidWorks Simulation (SolidWorks Corp.). The analysis was performed with the leaf spring having a radius of $0.1 \mathrm{~mm}$. Von Mises stress of the sensor was analyzed via the assumption that the perpendicular compressive load applied to the sensor top was $10 \mathrm{~N}$. Analytical results are shown in Figure 5. The results indicate that the load applied to the QCR was $9.42 \mathrm{~N}$ (it was calculated as $47.1 \mathrm{MPa}$ by multiplying the average stress on A-A', which has a section area of $0.2 \mathrm{~mm}^{2}$ ). Thus, the load transfer efficiency was found analytically to be 0.942 , which is $0.8 \%$ smaller than the value calculated using Eqs. (5)-(8). The maximum allowable load applied to the sensor is $31.8 \mathrm{~N}$ based on an allowable stress for AT-cut quartz crystals of $150 \mathrm{MPa}$.

\section{Fabrication}

The sensor was fabricated with the following procedure (Figure 6).

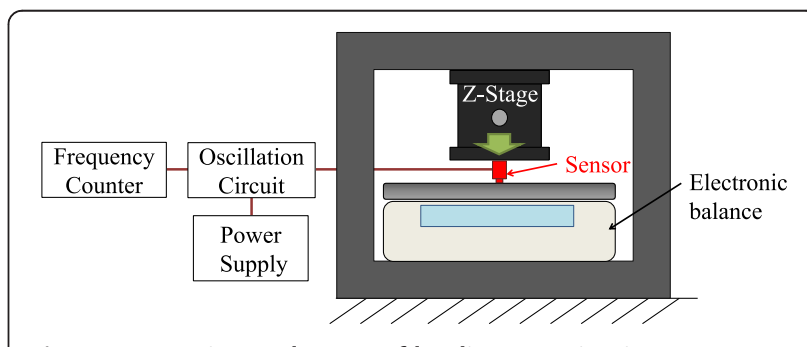

Figure 8 Experimental setup of loading examination. 


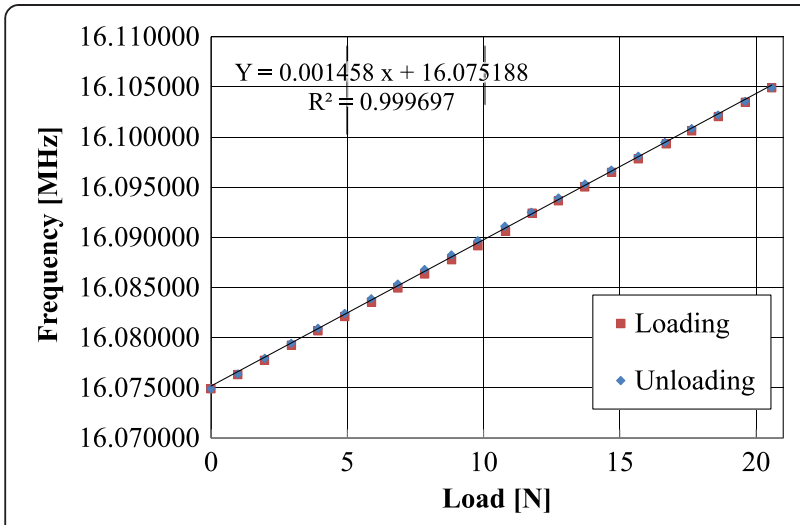

Figure 9 Result of the loading test.

\section{Fabrication of the QCR}

(a) Electrode patterns were formed to both faces of the AT-cut quartz crystal plate (thickness of $100 \mu \mathrm{m}$ ) using a photolithography technique lift-off process [20]. Chromium $(\mathrm{Cr})$ and gold $(\mathrm{Au})$ were deposited using sputtering equipment (E-200S, Canon ANELVA).

(b) Sheet resist (50X077, Nichigo-Morton Co.) was laminated to the quartz crystal.

(c) Sheet resist was patterned by photolithography.

(d) QCRs was formed into the pattern of sheet resist using sandblasting (Elfo-Blaster, Elfotec CO.).

\section{Fabrication of the Si structure}

(e) Photoresist (OFPR800 15CP,Tokyo Ohka Kogyo CO., LTD) was patterned on the Si wafer (thickness of $500 \mu \mathrm{m})$. (f) Silicon wafer was hollowed to a depth of approximately $50 \mu \mathrm{m}$ using deep reactive-ion etching (DRIE) (Multiplex-ASE-L, Sumitomo Precision Products Co.).

(g) Photoresist (SU-8 3025, MicroChem Corp.) was patterned on the Si wafer.

(h) Silicon wafer was formed into the pattern of SU-8 by DRIE, and SU-8 was removed.

\section{Assembly}

(i) Si structure was bonded from both sides of the QCR. Epoxy adhesive was used to bond the sensor parts, and wiring was attached using conductive silver paste.

A quartz crystal plate can be easily formed into any arbitrary shape using sandblasting. In addition, a Si wafer can be formed into minute structures having a high aspect ratio and high accuracy using DRIE.

\section{Results and discussion}

Load test

A fabricated sensor is shown in Figure 7. The volume of the sensor is $24.6 \mathrm{~mm}^{3}$ (width is $4 \mathrm{~mm}$, height is $5.6 \mathrm{~mm}$, depth is $1.1 \mathrm{~mm}$ ), which is drastically reduced relative to a conventional sensor [14]. We calibrated the frequency shift-applied load relationship of the fabricated QCR load sensor by loading test. A schematic of the calibration system is shown in Figure 8. In the experiment, an oscillation circuit and the sensor were connected, and the sensor output was measured using a frequency counter (53131A, Hewlett Packard). The QCR
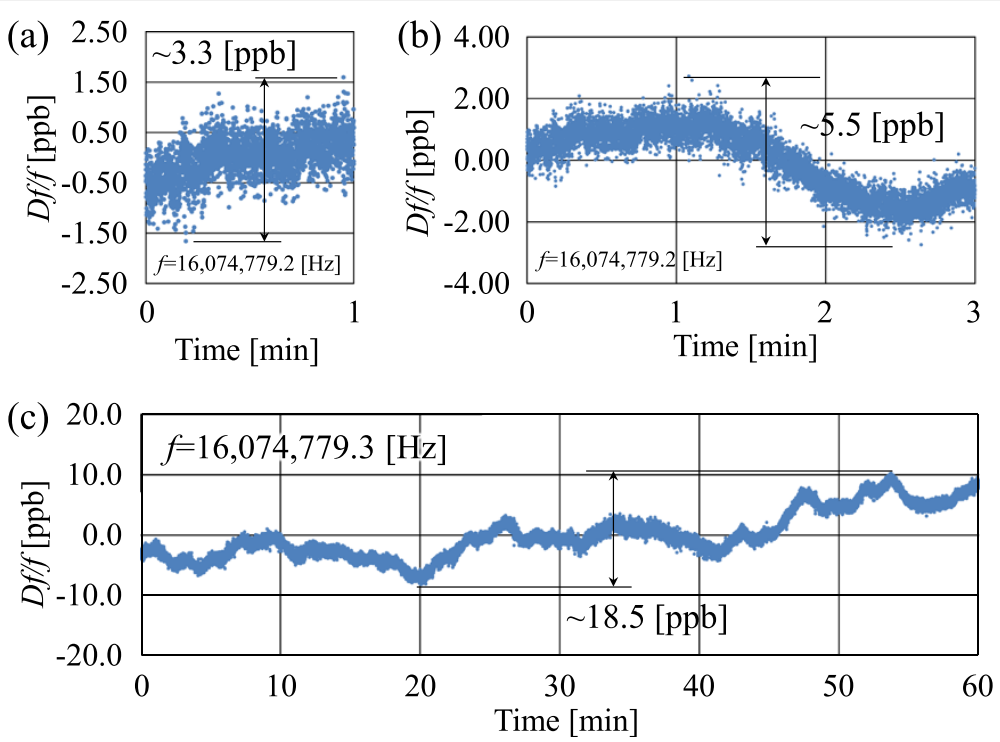

Figure 10 Stability of the sensor output for (a) 1 minute, (b) 3 minutes, (c) 1 hour. 
load sensor was fixed on the Z-stage, and electronic balance (UW4200A,SHIMADZU) was set under the sensor. The central axes of the sensor and the electronic balance were precisely aligned to avoid undesired lateral force to the sensor and to accurately measure the load applied to the sensor. Load was applied in $1 \mathrm{~N}$ increments from $0 \mathrm{~N}$ to $20 \mathrm{~N}$, and then decreased in $1 \mathrm{~N}$ increments from $20 \mathrm{~N}$ to $0 \mathrm{~N}$. The results are shown in Figure 9. The relationship between the applied load and the frequency shift was approximated by

$$
Y=1458 x \cdot 10^{-6}+16.075
$$

where, $x$ is the applied load $[\mathrm{N}]$ and $Y$ is the oscillating frequency $[\mathrm{MHz}]$. The correlation coefficient between the experimental results and the output of Eq. (9) is $\mathrm{R}^{2}=0.9997$. Thus, the results suggest that the fabricated QCR load sensor achieved a nearly linear relationship without preloading. The experimental results showed that the nonlinearity and hysteresis were $0.94 \%$ of the full scale (F.S.) and 1.68\% F.S., respectively. Additionally, sensitivity of the sensor was $1458 \mathrm{~Hz} / \mathrm{N}$, which is 2.5 times greater than that of the conventional sensor $(573 \mathrm{~Hz} / \mathrm{N}[14])$.

\section{Stability of sensor output}

Time stability of the sensor output was measured. The measurement was conducted after the sensor output reached steady state at a controlled temperature $\left(28^{\circ} \mathrm{C}\right)$. Figure 10 (a), (b), and (c) show fluctuation of the sensor output for $1 \mathrm{~min}, 3 \mathrm{~min}$, and $1 \mathrm{~h}$, respectively. Here, $f$ and $D f$ shows the average value of the measurement frequency and difference value between $f$ and measured frequency, respectively. Therefore, $D f / f$ shows relative error in each measurement time. From the results, the frequency fluctuations of the fabricated sensor were $3.3 \mathrm{ppb}$ for $1 \mathrm{~min}, 5.5 \mathrm{ppb}$ for $3 \mathrm{~min}$, and $18.5 \mathrm{ppb}$ for $1 \mathrm{~h}$, respectively. The sensor output showed high stability, even over the

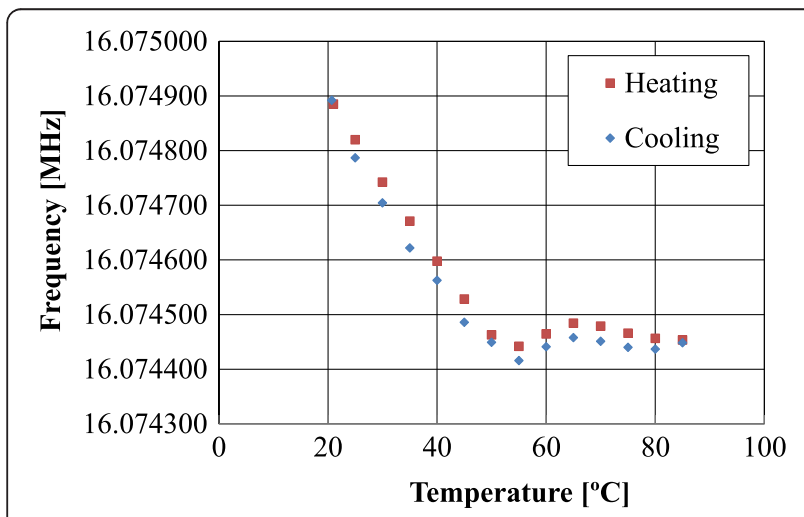

Figure 11 Temperature characteristics $\left(20\right.$ to $\left.80^{\circ} \mathrm{C}\right)$.

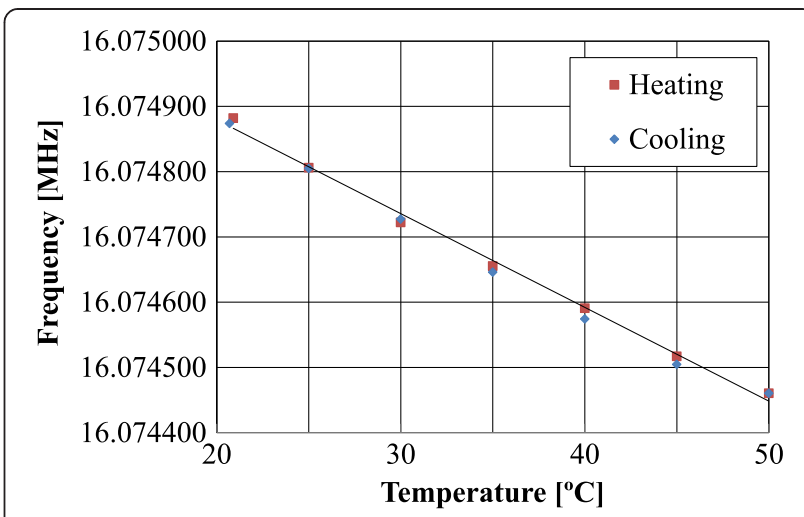

Figure 12 Temperature characteristics $\left(20\right.$ to $\left.50^{\circ} \mathrm{C}\right)$.

course of a long-term measurement. The fluctuation of the external load estimated from the fluctuation of the sensor output was $0.04 \mathrm{mN}$ for $1 \mathrm{~min}, 0.06 \mathrm{mN}$ for $3 \mathrm{~min}$, and $0.20 \mathrm{mN}$ for $1 \mathrm{~h}$, respectively. Compared to the conventional sensor (3.21 mN [14]), the fabricated sensor had superior stability with regard to the sensor output.

\section{Temperature characteristics}

The sensor output was approximated via the differential change in temperature. The sensor output was measured when the sensor was heated in $5^{\circ} \mathrm{C}$ increments from $20^{\circ} \mathrm{C}$ to $80^{\circ} \mathrm{C}$ and cooled in $5^{\circ} \mathrm{C}$ increments from $80^{\circ} \mathrm{C}$ to $20^{\circ} \mathrm{C}$. The fabricated sensor including the oscillation circuit was placed in the thermostat chamber; the results are shown in Figure 11. The relationship between the temperature and sensor output was nonlinear and had apparent hysteresis. This might have been caused by the temperaturedependent change of the Young's modulus of the epoxy resin used for bonding. Here, the temperature and the sensor output are linear in the range from $20^{\circ} \mathrm{C}$ to $50^{\circ} \mathrm{C}$. Thus, the sensor output was measured when temperature of the sensor was varied from $20^{\circ} \mathrm{C}$ to $50^{\circ} \mathrm{C}$. The results are shown in Figure 12. The relationship between temperature and the sensor output was approximated by

$$
Y=-14.363 T \cdot 10^{-6}+16.075
$$

where, $T$ is the temperature of the sensor $\left[{ }^{\circ} \mathrm{C}\right]$ and $Y$ is the oscillation frequency $[\mathrm{MHz}]$. The correlation coefficient

Table 1 Comparison of sensor characteristics

\begin{tabular}{llll}
\hline & $\begin{array}{l}\text { Size }[\mathrm{mm}] \\
\left(\text { Volume }\left[\mathrm{mm}^{3}\right]\right)\end{array}$ & $\begin{array}{l}\text { Sensitivity } \\
{[\mathrm{Hz} / \mathrm{N}]}\end{array}$ & $\begin{array}{l}\text { Stability of sensor } \\
\text { output for } \mathbf{3} \text { min }[\mathrm{mN}]\end{array}$ \\
\hline $\begin{array}{l}\text { A. Asakura } \\
\text { et al. [13] }\end{array}$ & $\varphi 13 \times 13(1724.6)$ & 360 & 22.5 \\
$\begin{array}{l}\text { K. Narumi } \\
\text { et al. [14] }\end{array}$ & $\varphi 7 \times 11(423.1)$ & 573 & 3.21 \\
$\begin{array}{l}\text { Fabricated } \\
\text { sensor }\end{array}$ & $4 \times 5.6 \times 1.1(24.6)$ & 1458 & 0.06 \\
\hline
\end{tabular}


between the experimentally measured and analytically calculated results is $R_{2}=0.995$. Furthermore, the results suggest that the temperature and the sensor output follow a linear relation within the range of $20^{\circ} \mathrm{C}-50^{\circ} \mathrm{C}$. Temperature compensation can be easily implemented via integration of a temperature sensor with the QCR load sensor, following the relationship given by Eq. (10).

\section{Discussion}

Table 1 shows a comparison of the characteristics of the fabricated QCR load sensor to conventional QCR load sensors. The fabricated sensor was successfully miniaturized and its sensitivity and stability were improved. In this paper, we used an adhesive to bond the sensor components together. Because the melting point of the adhesive was relatively low, it may have affected the linearity of the sensor output with regard to temperature changes. If the $\mathrm{Si}$ parts and the quartz crystal plate were to be bonded using hybrid plasma bonding [21], the thermal properties of the sensor would be improved. Our future studies will investigate superior bonding conditions for the sensor.

\section{Conclusions}

In this paper, we proposed a novel design and fabrication method for a QCR load sensor. Using microfabrication and bonding, the assembly process was simplified, and we achieved miniaturization of the QCR load sensor. The proposed retention mechanism has improved load transfer efficiency via the Si structure formed using DRIE. We successfully fabricated a high-sensitivity load sensor, and significant miniaturization of the sensor was realized using microfabrication and bonding techniques. The parts all have a flat structure and the fabrication process is based on photolithography, and therefore, is suitable for mass production.

In the future, we will work toward further improvements regarding the sensitivity and miniaturization of the sensor. A key issue will be the methods used to bond the quartz crystal plate and Si parts together.

\section{Abbreviations}

QCR: Quartz crystal resonator; DRIE: Deep reactive ion etching.

\section{Competing interests}

The authors declare that they have no competing interests.

\section{Authors' contributions}

All authors equally contributed. All authors read and approved the final manuscript.

\section{Acknowledgement}

This work was partially supported by Center of Innovation Program and A-STEP, JST.

\section{References}

1. da Silva JG, de Carvalho AA, da Silva DD (2002) A strain gauge tactile sensor for finger-mounted applications. IEEE Trans Instrum Meas 51:18-22

2. Stef DM, Farca A (2012) Strain gauge force transducer and virtual instrumentation used in a measurement system for retention forces of palatal plates or removable dentures. IEEE Sens J 12:2968-2973

3. Itoh T, Suga T (1994) Piezoelectric force sensor for scanning force microscopy. Sens Actuators A Phys 43:305-310

4. Motoo K, Arai F, Fukuda T (2007) Piezoelectric vibration-type tactile sensor using elasticity and viscosity change of structure. IEEE Sens J 7(7):1044-1051

5. Resolution N, Beyeler F, Muntwyler S, Nelson BJ, Member S (2009) A six-axis MEMS force - Torque sensor with micro-newton and nano-newtonmeter resolution. J Microelectromech S 18:433-441

6. Ballato A, Bechman R (1960) Effect of initial stress in vibrating quartz plates. Proc IRE 48:261-262

7. Ratajski J (1968) Force frequency coefficient of singly rotated vibrating quartz crystals. IBM J Dev Res 12:92-99

8. Jorgensen JD (1978) Compression mechanisms in a-quartz structures- $\mathrm{SiO}_{2}$ and $\mathrm{GeO}_{2}$. J Appl Phys 49:5473-5478

9. Atkinson BK (1980) Stress corrosion and the rate-dependent tensile failure of a fine-grained quartz rock. Techtonophysics 65:281-290

10. Dong YG, Wang JS, Feng GP, Wang XH (1999) Self-Temperature-testing of the quartz resonant force sensor. IEEE Trans Instrum 48(6):1038-1040

11. EerNisse EP (2001) Review of thickness-shear mode quartz resonator sensors for temperature and pressure. IEEE Sens J 1(1):79-87

12. Wang Z, Zhu H, Dong Y, Feng G (2003) A thickness-shear quartz force sensor with dual-mode temperature compensation. IEEE Sens J 3(4):490-497

13. Asakura A, Fukuda T, Arai F (2009) Design, fabrication and characterization of compact force sensor using AT-cut quartz crystal resonators. J Robot Mechatron 21(2):260-266

14. Narumi K, Asakura A, Fukuda T, Arai F (2010) Design and characterization of load sensor with AT-Cut QCR for miniaturization and resolution improvement. J Robot Mechatron 22(3):286-292

15. Wu B, Kumar A, Pamarthy S (2010) High aspect ratio silicon etch: a review. J Appl Phys 108:051101

16. Martya F, Rousseaua L, Saadanya B, Merciera B, Francaisa O, Mitab Y, Bourouina $T$ (2005) Advanced etching of silicon based on deep reactive ion etching for silicon high aspect ratio microstructures and three-dimensional micro- and nanostructures. Microelectron J 36:673-677

17. Stevens DS, Tiersten HF, Sinha BK (1983) Temperature dependence of the resonant frequency of electrode contoured AT-cut quartz crystal resonators. J Appl Phys 54:1704-1716

18. Wang J, Yu JD, Yong YK, Imai T (2008) A finite element analysis of frequency-temperature relations of AT-cut quartz crystal resonators with higher-order Mindlin plate theory. Acta Mech 199:117-130

19. Muraoka S, Nishimura H (1996) Characteristics of a rectangular AT cut quartz resonator as a force sensor. Coll Pap Soc Instrum Control Eng 32(4):604-606

20. Kayaku M (2014) LOR and PMGI Resists. http://microchem.com/pdf/PMGIResists-data-sheetV-rhcedit-102206.pdf. Accessed 15 Jan 2014

21. Howlader MMR, Suehara S, Takagi H, Kim TH, Maeda R, Suga T (2006) Roomtemperature microfluidics packaging using sequential plasma activation process. IEEE Trans Adv Packag 29(3):448-456

\section{doi:10.1186/s40648-014-0003-x}

Cite this article as: Murozaki et al:: Miniaturized load sensor using quartz crystal resonator constructed through microfabrication and bonding. ROBOMECH Journal 2014 1:3. 